\title{
Polymorbidity as a Challenge to Health Services in the XXI Century
}

\author{
Sevostyanova $\mathrm{EV}^{*}$ and Nicolaev $\mathrm{YuA}$ \\ Federal Research Center of Fundamental and Translational Medicine, Russia \\ *Corresponding author: Sevostyanova EV, Federal Research Center of Fundamental and Translational Medicine, \\ 630117, Novosibirsk, Russia
}

\begin{tabular}{|c|c|}
\hline ARTICLE INFO & ABSTRACT \\
\hline Received: 慧 April 06, 2020 & Citation: Sevostyanova EV, Nicolaev YuA. Polymorbidity as a Challenge to Health \\
\hline Published: 慧 April 16, 2020 & Services in the XXI Century. Biomed J Sci \& Tech Res 27(1)-202 \\
\hline
\end{tabular}

\section{Opinion}

In recent decades, the widespread growth of combined pathology, which significantly affects the course and outcome of diseases, has become a serious medical-social and organizationaleconomic problem. Currently, combined pathology is considered within the framework of the concept of multimorbidity. Multimorbidity is defined as the presence of two and / or more chronic diseases pathogenetically interconnected and / or coinciding in time in one patient, regardless of the activity of each of them. Recent epidemiological studies have convincingly shown a high prevalence of multimorbidity around the world, associated, also, with an aging population and an increase in life expectancy. Multimorbidity has adverse medical and social consequences. The interaction of combined diseases changes the classical clinical picture, the nature of the course of diseases, increases the number of complications and their severity, worsens the quality of life and life prognosis. In patients with multimorbidity, the diagnosis, choice of tactics and means of treatment are complicated, financial costs increase, or when following the standards of mandatory medical care, patients of this category do not achieve the target results. All this leads to a decrease in the effectiveness of treatment, a decrease in patient adherence to therapy, polypharmacy, a decrease in the quality of life, and increased mortality rates.

Multimorbidity is a heavy burden for the economies of countries. In the presence of multimorbidity, the costs of diagnosing and treating diseases significantly increase, and the volume and cost of providing medical care increases. The syntropy of nosologies must be considered for targeted prevention, adequate treatment and prediction of complications. At the same time, it should be noted that today the majority of studies are mainly mononosological in nature and most guidelines are developed for individual diseases, which represents the greatest difficulties in managing patients with multimorbidity. Lack of knowledge in this area leads to defects in the organization of medical care. To develop effective strategies for the prevention and treatment of diseases of a multimorbid patient, it is necessary to study the features, structure, patterns of multimorbidity and risk factors for its formation, as well as the identification of common pathogenetic links that underlie its formation. There are works indicating the influence of age, gender, and also some socio-economic factors (income level, educational level) on multimorbidity patterns. Along with this, it also seems reasonable to study the role of other modifiable hemodynamic and metabolic risk factors of chronic non communicable diseases in the formation of multimorbidity, which include: high blood pressure, obesity, dyslipidemia, hyperglycemia, hyperuricemia, etc. Together, the considered risk factors can be considered as manifestations of the metabolic syndrome, the presence of which significantly increases the risk of developing multiple diseases, primarily diseases of the cardiovascular, endocrine systems, digestive system, and also the musculoskeletal system.

Moreover, the features of the influence of these risk factors on the formation of multimorbidity in different regions that differ in their climatic and geographical and socio-economic characteristics remain unexplored. At the same time, epidemiological data on the prevalence of multimorbidity in different geographical zones vary significantly. In addition, there are pronounced differences in the morbidity and mortality rates in different regions, which 
determine the need to study the regional characteristics of multimorbidity and its determining factors. When searching for common pathogenetic links of the combined diseases that underlie the formation of multimorbidity, it seems to us promising to study such general pathological processes as an imbalance of neurohumoral regulatory mechanisms, features of the course of chronic systemic inflammation, oxidative stress and impaired antioxidant defense, circulatory disorders in organs and the formation of endothelial dysfunction, psychoemotional stress, followed by vegetative and metabolic disorders. To our opinion, based on the studies conducted, there is reason to believe that the pathogenetic relationships of inflammation, metabolic disorders, first of all, lipid and carbohydrate metabolism disorders, oxidative stress can be considered as one of the main pivots of multimorbid pathology.

At the same time, atherosclerotic vascular damage, endothelial dysfunction, leading to microcirculatory disorders, ischemia and

ISSN: 2574-1241

DOI: 10.26717/BJSTR.2020.27.004446

Sevostyanova EV. Biomed J Sci \& Tech Res

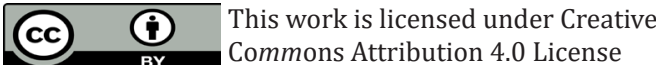

Submission Link: https://biomedres.us/submit-manuscript.php dysfunction of the internal multiple organs, can be considered as a unifying pathological process in the formation of multimorbidity. It is necessary to develop clinical recommendations for diagnosis, treatment and rehabilitation in the presence of multimorbidity, special treatment and rehabilitation programs for the management of a multimorbid patient.The development of suchrecommendations and programs should be based on the identification of common risk factors, pathogenetic links and common key points in the formation and course of combined pathology, which would exclude polypharmacy and unreasonable interventions. Due to the prevalence and socio-economic consequences of multimorbidity, this problem poses a challenge to health services in the $21^{\text {st }}$ century, requiring joint effective efforts of specialists from different countries.

$\begin{array}{ll}\text { BIOMEDICAL } & \text { Assets of Publishing with us } \\ \text { RESEARCHES } & \text { - Global archiving of articles } \\ & \text { - Immediate, unrestricted online access } \\ & \text { - Rigorous Peer Review Process } \\ & \end{array}$

